\title{
Salvage Surgery for Initially Unresectable Locally Advanced Hepatocellular Carcinoma Downstaged by Hepatic Arterial Infusion Chemotherapy
}

\author{
YUICHI GOTO, TORU HISAKA, HISAMUNE SAKAI, KATSUAKI TAKAGI, \\ SHOGO FUKUTOMI, YOSHITO AKAGI and KOJI OKUDA \\ Department of Surgery, Kurume University School of Medicine, Kurume, Japan
}

\begin{abstract}
Background/Aim: Recent studies have demonstrated the efficacy of salvage surgery following downstaging of hepatocellular carcinoma (HCC). The aim was to assess the outcomes of salvage surgery after successful downstaging using hepatic arterial infusion chemotherapy (HAIC). Patients and Methods: Patients whose diagnosis was unresectable locally advanced HCC and who were resected after conversion to a resectable status by HAIC were included. The overall survival (OS) rate, and disease-free survival (DFS) rate were analyzed by stratifying patients into those with Vp3/4, Vv2/3, and those without major vascular invasion (MVI). Results: Eighteen patients were censored. Among them, six patients had Vp3/4, four patients had Vv2/3, and eight patients had no MVI. The 5year OS rates of patients with Vp3/4 and those without MVI were $83 \%$ and $73 \%$, respectively, whereas those with $V v 2 / 3$ had $0 \%(p<0.001)$. Conclusion: Salvage surgery has the potential to provide excellent outcomes in resectable HCC patients, except for those with $\mathrm{Vv} 2 / 3$.
\end{abstract}

Among patients with unresectable locally advanced hepatocellular carcinoma (HCC), some patients can be converted to be technically resectable, although no consensus exists regarding the optimal therapeutic strategy for these patients. In clinical practice, downstaging followed by salvage surgery is performed for some of these patients, and some researchers have reported favorable outcomes (1-8). The aim of this study was to analyze the survival benefit of

This article is freely accessible online.

Correspondence to: Yuichi Goto, MD, Ph.D., Department of Surgery, Kurume University School of Medicine, 67 Asahi-machi, Kurume 830-0011, Japan. Tel: +81 942353311 ext. 3541, Fax: +81 942358967,e-mail: gotou_yuuichi@med.kurume-u.ac.jp

Key Words: Downstaging, hepatocellular carcinoma, low dose FP, salvage surgery. downstaging with hepatic arterial infusion chemotherapy (HAIC) followed by salvage surgery for initially unresectable locally advanced HCC by stratifying patients according to the presence or not of major vascular invasion (MVI).

\section{Patients and Methods}

Study population. Patients who underwent hepatectomy for HCC at our institution between 1999 and 2013 were identified. Among these patients, those whose diagnoses were initially unresectable HCC due to locally advanced disease and were resected after successful downstaging using HAIC were included. We divided patients into three groups by tumor extent at initial diagnosis: tumors with $\mathrm{Vp} 3$ (first-order branches of the portal vein)/Vp4 (main trunk or opposite first branch of the portal vein) vascular invasion (Group A), tumors with Vv2 (main trunk of the hepatic vein)/Vv3 [inferior vena cava (IVC) or right atrium (RA)] vascular invasion (Group B), and tumors without MVI (Group C), which includes tumors without $\mathrm{Vp} 3 / 4$ or $\mathrm{Vv} 2 / 3$ vascular invasion. Overall survival (OS) rates, disease-free survival (DFS) rates, and patterns of recurrence were analyzed by reviewing medical record. This study was conducted in accordance with the Declaration of Helsinki and the ethical guidelines for clinical studies, and the ethics committee of our institution approved this research (No. 17149).

Hepatic arterial infusion chemotherapy. HAIC was performed after placement of an injection port implanted in a subcutaneous pocket. The tip of the catheter was placed at the common hepatic artery or proper hepatic artery. Low-dose 5-FU/cisplatin (FP) via the injection port was used as the HAIC regimen (9). The first course of chemotherapy consisted of daily administration of cisplatin $\left(7 \mathrm{mg} / \mathrm{m}^{2}\right)$ followed by 5 -FU $\left(170 \mathrm{mg} / \mathrm{m}^{2}\right)$ on days 1 to 5 , for 1 and $5 \mathrm{~h}$, respectively, by mechanical infusion pump. After cessation of chemotherapy on days 6 and 7, patients received an additional 4 cycles of chemotherapy to complete induction chemotherapy. One or two months after the initial four cycles of induction HAIC, patients received an additional 2 or 4 cycles of low-dose FP based on tumor regression and/or complications; this was repeated continuously until disease progression (9). The response to HAIC was evaluated by CT after 4 cycles of HAIC and every 2 months according to the modified Response Evaluation Criteria in Solid Tumors (10). Senior surgeons assessed resectability during multidisciplinary meetings. 
Table I. Baseline patient characteristics.

\begin{tabular}{|c|c|c|c|}
\hline Variables & Pre-chemotherapy & Pre-hepatectomy & $p$-Value \\
\hline Age, years, median (range) & $68.5(46-79)$ & & \\
\hline Gender, male, n (\%) & $12(66)$ & & \\
\hline \multicolumn{4}{|l|}{ Etiologies, $\mathrm{n}(\%)$} \\
\hline $\mathrm{HCV} / \mathrm{HBV} /$ Non-B, -C & $9(50) / 5(28) / 4(22)$ & & \\
\hline Child-Pugh score 5/6 (\%) & $18(100) / 0(0)$ & $16(89) / 2(11)$ & 0.089 \\
\hline ICG-R15 (\%), median (range) & & $20.2(6.1-49.9)$ & \\
\hline $\operatorname{AFP}(\mathrm{ng} / \mathrm{ml})$, median (range) & $3748(17.8-270,000)$ & $31.8(2.7-10,452)$ & 0.026 \\
\hline PIVKA-II (mAU/ml), median (range) & $3106(81-32,200)$ & $210(5-22,456)$ & 0.041 \\
\hline Tumor size, $\mathrm{cm}$, median (range) & $71(15-150)$ & $56(5-85)$ & 0.012 \\
\hline Tumor size $>10 \mathrm{~cm}, \mathrm{n}(\%)$ & $5(28)$ & $0(0)$ & 0.005 \\
\hline Tumor size reduction rate, $\mathrm{n}(\%)$ & & $32.2(13.3-66.7)$ & \\
\hline Tumor number, median (range) & $2(1-5)$ & $1.5(1-3)$ & 0.328 \\
\hline Single, $\mathrm{n}(\%)$ & $7(39)$ & $9(50 \%)$ & 0.502 \\
\hline Multiple, n (\%) & $11(61)$ & $9(50 \%)$ & 0.502 \\
\hline \multicolumn{4}{|l|}{ T stage* } \\
\hline $\mathrm{T} 2 / \mathrm{T} 3 \mathrm{a} / \mathrm{T} 3 \mathrm{~b}, \mathrm{n}(\%)$ & $1(5) / 7(39) / 10(56)$ & $4(22) / 4(22) / 10(56)$ & 0.252 \\
\hline Macroscopic PVTT, n (\%) & $9(50)$ & $9(50)$ & 0.252 \\
\hline $\mathrm{vp} 2 / \mathrm{vp} 3 / \mathrm{vp} 4, \mathrm{n}(\%)$ & $3(17) / 4(22) / 2(11)$ & $3(17) / 5(26) / 1(5)$ & 0.798 \\
\hline Macroscopic HVTT, n (\%) & $4(22)$ & $4(22)$ & \\
\hline $\mathrm{vv} 2 / \mathrm{vv} 3, \mathrm{n}(\%)$ & $1(5) / 3(17)$ & $2(11) / 2(11)$ & 0.462 \\
\hline
\end{tabular}

HCV: Hepatitis C virus; HBV: hepatitis B virus; ICG-R15: indocyanine green retention rate at 15 min; AFP: $\alpha$-fetoprotein; PIVKA-II: protein induced by vitamin K absence or antagonist-II; PVTT: portal vein tumor thrombus; HVTT: hepatic vein tumor thrombosis. *According to the American Joint Committee on Cancer $7^{\text {th }}$ TNM classification.

Surgical treatment and technique. The objective of surgery was to resect all detectable lesions with tumor-free margins. In patients with multiple bilateral tumors that could not be resected by a single hepatectomy and for lesions $<2 \mathrm{~cm}$, concomitant radiofrequency ablation therapy (RFA) was performed. Thrombectomy was performed in patients with Vp4 vascular invasion under temporary clamping of the main portal vein. Among patients with Vv3 vascular invasion, in case the tumor thrombosis was located in the IVC, a total hepatic venous exclusion procedure (THVE) was applied (11). In case Vv3 vascular invasion extended into the RA, the patient was placed on cardiopulmonary bypass and a longitudinal incision was made from the RA to the IVC under cardiac arrest and THVE, and then the tumor thrombosis was removed under direct view.

Statistical analysis. Continuous variables were expressed as median (range). Student's $t$-test, $\chi^{2}$ test, or Fisher's exact test was used where appropriate to compare the clinical parameters between the two groups. Survival data were analyzed using the Kaplan-Meier method. Survival curves were compared using the log-rank test. OS was calculated from the date of initial HAIC until death or last follow-up. DFS was calculated from the date of hepatectomy until recurrence or last followup. For all tests, $p<0.05$ was defined as indicating a statistically significant difference. Statistical analysis was performed using the JMP version 13.0 software program (SAS Institute, Cary, NC, USA).

\section{Results}

Patients and tumor characteristics before and after HAIC. Between 1999 and 2013, 1,020 patients with HCC underwent hepatic resection at our institution. Among these, 18 patients underwent resection after successful downstaging using HAIC. Patients were classified as having Vp3/4 (Group A; n=6), Vv2/3 (Group B; n=4), or no MVI (Group C; n=8) at diagnosis. Clinical and pathological patient characteristics are listed in Table I. The median tumor size was $71 \mathrm{~mm}$ (range $=15-150 \mathrm{~mm}$ ) and 5 patients $(28 \%)$ had tumors $>100$ $\mathrm{mm}$; however, the median tumor size decreased to $56 \mathrm{~mm}$ (range $=5-85 \mathrm{~mm})$ after HAIC $(p=0.012)$. Eleven patients $(61 \%)$ had multiple tumors before treatment and nine patients $(50 \%)$ had multiple tumors after HAIC $(p=0.328)$. The median levels of both tumor markers, AFP and PIVKAII, were elevated at $3,748 \mathrm{ng} / \mathrm{ml}($ range $=17.8-270,000 \mathrm{ng} / \mathrm{ml})$ and 3,106 $\mathrm{mAU} / \mathrm{ml}$ (range=81-3,220 $\mathrm{mAU} / \mathrm{ml}$ ), respectively, at diagnosis, but they decreased to $31.8 \mathrm{ng} / \mathrm{ml}$ (range=2.7$10,452 \mathrm{ng} / \mathrm{ml}$ ) and $210 \mathrm{mAU} / \mathrm{ml}$ (range=5.0-22,456 $\mathrm{mAU} / \mathrm{ml})$, respectively, after HAIC ( $p=0.026$ and 0.041 , respectively). All patients in Groups A and B had tumor thrombosis shrinkage, whereas in 2 patients (11\%), regression of tumor thrombosis from $\mathrm{Vp} 4$ to $\mathrm{Vp} 3$ and $\mathrm{Vv} 3$ to $\mathrm{Vv} 2$, respectively, was observed.

Hepatic arterial infusion chemotherapy. The modality, duration, and efficacy of HAIC are summarized in Table II. All patients received low-dose FP, and 8 of 18 patients (44\%) underwent additional TACE. The mean period of chemotherapy was 5.1 months (range=1.0-25.4 months). Fifteen patients $(83 \%)$ achieved a PR and were converted to 
Table II. Downstaging chemotherapy and operative findings.

\begin{tabular}{lcc}
\hline Variables & $\begin{array}{c}\text { Median } \\
\text { (range) }\end{array}$ & $\begin{array}{c}\text { Number of } \\
\text { patients (\%) }\end{array}$ \\
\hline Additional TACE & & $8(44)$ \\
Duration of chemotherapy, months & $5.2(1-25)$ & \\
Objective response & & $15(83) / 3(17)$ \\
PR/SD & & $1(5)$ \\
Type of resection & $1(5)$ \\
Right tri sectionectomy & & $6(33)$ \\
Extended right hepatectomy & & $1(5)$ \\
Right hepatectomy & & $2(17)$ \\
Left tri sectionectomy & & $1(5)$ \\
Extended left hepatectomy & & $1(5)$ \\
Left hepatectomy & & $2(10)$ \\
Extended posterior sectionectomy & & \\
Anterior sectionectomy & & \\
Segmentectomy & & \\
Resection margin, mm & $12.7(1-25)$ & \\
Operation time, min & $500(380-1,080)$ & \\
Amount of bleeding, ml & $1,240(230-9,115)$ & \\
\hline
\end{tabular}

*p-Value: pre-downstaging chemotherapy $v s$. post-downstaging chemotherapy.

resectable status. In addition, 3 patients with stable disease (SD), even after HAIC, experienced some degree of tumor shrinkage and were also converted to resectable status.

Surgical procedures. Overall, 14 of 18 patients underwent major hepatectomy. Concomitant RFA was performed in 1 patient. In 9 patients, tumor thrombectomy was necessary. Extracorporeal circulation was required in 1 case with RA tumor thrombosis and THVE was necessary in 1 case with IVC tumor thrombosis. The median operation time was 500 min (range $=380-1,080$ min) with a median amount of bleeding of 1,240 $\mathrm{ml}$ (range=230-9,115 ml). All patients achieved macroscopic $\mathrm{R} 0$ resection (Table II).

Short- and long-term outcomes. All patients were discharged without in-hospital mortality. OS and DFS of patients are shown in Figure 1. The median observation time was 58.5 months (range=9.5-122.6 months). The 1-, 3-, and 5-year OS rates were $94.4 \%, 83.3 \%$, and $58.7 \%$, respectively. The 1-, 3-, and 5-year DFS rates were $61.1 \%, 33.3 \%$, and $33.3 \%$, respectively. OS by different patient groups is shown in Figure 2. The 1-, 3-, and 5year OS rates in Group A were 100\%, 100\%, and 83\%, respectively, and those in Group C were $100 \%, 100 \%$, and $73 \%$, respectively. The 1-, 3-, and 5-year OS rates in Group B were $75 \%, 50 \%$, and $0 \%$, respectively. DFS of downstaged patients by group are shown in Figure 3. The 1-, 2-, and 3-year DFS rates in Group A were $100 \%, 33 \%$, and 33\%, respectively and those in Group C were $62 \%, 50 \%$, and $50 \%$, respectively. With respect to DFS in Group B, all patients experienced recurrences within 1 year. The OS and DFS rates in Group B were worse

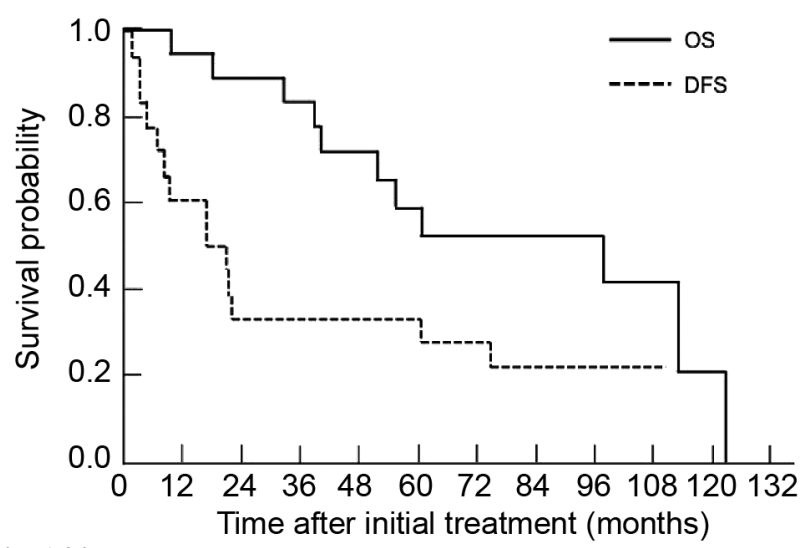

No. at risk

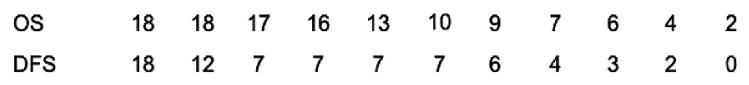

Figure 1. Overall survival and disease-free survival of downstaged patients $(n=18)$.

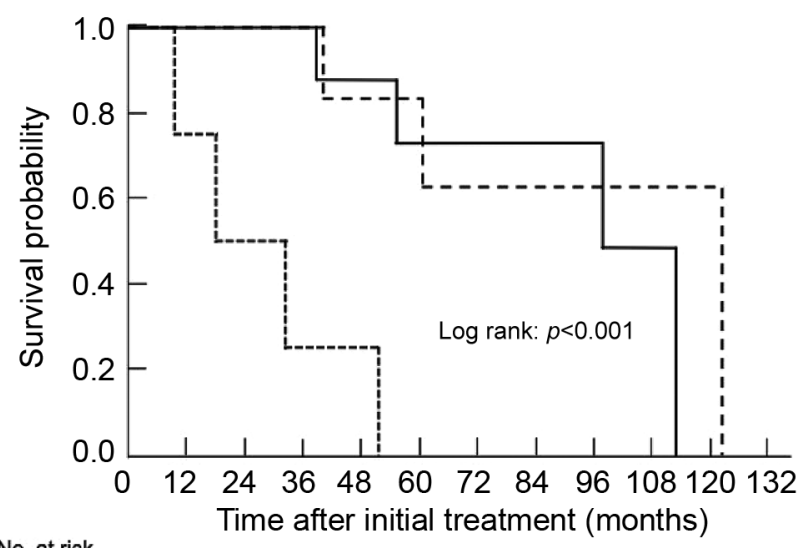

No. at risk

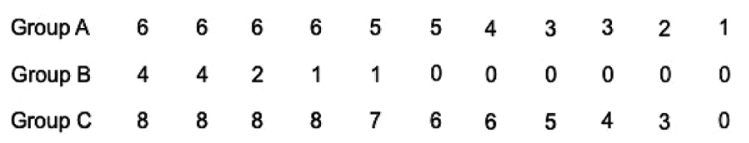

$$
\begin{aligned}
& ---V p 3 / 4 \text { vascular invasion } \\
& ---V_{v 2} / 3 \text { vascular invasion } \\
& \text { - Without major vascular invasion }
\end{aligned}
$$

Figure 2. Overall survival rate by stratifying patients into those with Vp3/4, Vv2/3, and those without major vascular invasion. $p<0.001$ by log-rank test.

compared to those in Groups A and C $(p<0.001$ and $p<0.001$, respectively). Overall, 14 of 18 patients (78\%) experienced postoperative recurrences. In Group A, 1 patient had metastasis to the bone and 4 patients had a single intrahepatic recurrence. 


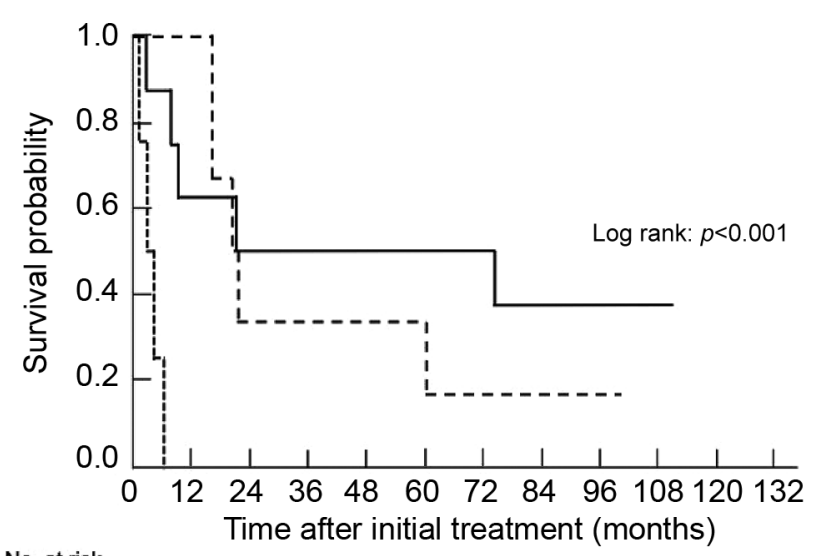

No. at risk

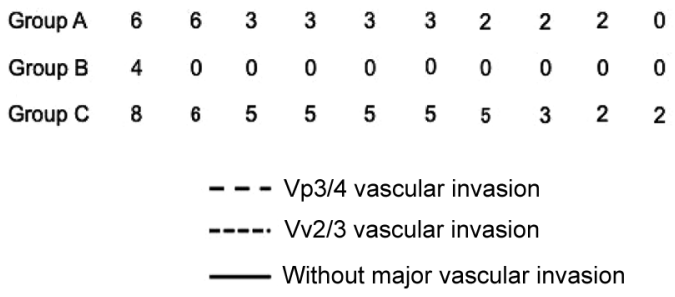

Figure 3. Disease-free survival rate by stratifying patients into those with Vp3/4, Vv2/3, and those without major vascular invasion. $p<0.001$ by log-rank test.

In Group B, 2 patients had metastasis to the lung, 1 patient had lung metastases accompanying by relapsed RA tumor thrombosis, and 1 patient had multiple intrahepatic recurrences. In Group C, 2 patients had metastasis to the lung and 3 patients had intrahepatic recurrences (Table III).

\section{Discussion}

We assessed the outcomes of salvage surgery in 18 patients who were successfully converted from unresectable to resectable status with HAIC using a low-dose FP regimen. Patients had different degrees of tumor extent, including $\mathrm{Vp} 3 / 4, \mathrm{Vv} 2 / 3$, and no MVI. Analysis of patients with different tumor characteristics demonstrated excellent outcomes especially in patients with $\mathrm{Vp} 3 / 4$ and those without MVI. There is no consensus about how to treat locally advanced HCC patients who respond well to chemotherapy and who have been converted technically from unresectable to resectable. In clinical practice, it is always difficult to decide whether these patients should be resected or not resected. However, our series of patients achieved a 5-year OS of $58.7 \%$ even though these patients were highly selected during chemotherapy. What is also interesting is that the OS and DFS rates of these two groups were almost the same even though their oncological malignant potential would be different. These results suggest that salvage surgery has the potential to provide excellent outcomes and a chance of cure by selecting
Table III. Site of recurrence after surgery and tumor characteristics.

\begin{tabular}{lcccc}
\hline Site of recurrence & $\begin{array}{c}\text { Total } \\
\text { number }\end{array}$ & $\begin{array}{c}\text { Group } \\
\text { A }\end{array}$ & $\begin{array}{c}\text { Group } \\
\text { B }\end{array}$ & $\begin{array}{c}\text { Group } \\
\text { C }\end{array}$ \\
\hline Intrahepatic & 8 & 4 & 1 & 3 \\
Single & 7 & 4 & 0 & 3 \\
Multiple & 1 & 0 & 1 & 0 \\
Extrahepatic & 7 & 1 & 4 & 2 \\
Lung & 5 & 0 & 3 & 2 \\
Bone & 1 & 1 & 0 & 0 \\
RA tumor thrombus & 1 & 0 & 1 & 0 \\
\hline
\end{tabular}

RA: Right atrium.

patients during the chemotherapy period even if they were accompanied by major portal invasion or not. In contrast, OS and DFS of patients with Vv2/3 were dismal compared to those of other patients even after effective downstaging using HAIC. These patients had a high incidence of extrahepatic recurrence during the very early postoperative period, within 12 months after surgery, which affected OS. The existence of $\mathrm{Vv} 2 / 3$ is reported to be one of the poorest prognostic factors, with no established therapeutic strategies $(12,13)$. The reported median survival time (MST) of these patients who did not undergo surgical intervention has been reported to range from 5.5 to 9.5 months $(12,13)$. These patients have also a risk of sudden death caused by pulmonary embolism from the tumor thrombosis, especially patients with tumor thrombosis in the RA (12). These risks may justify surgical intervention even in patients with $\mathrm{Vv} 2 / 3$.

In the recent literature, downstaging followed by salvage surgery has been performed after multidisciplinary therapy including transarterial chemoembolization (TACE) $(6,7)$, HAIC $(1,2)$, immunotherapy (14), chemoradiotherapy $(3,4)$, and systemic chemotherapy $(5,8)$. The reported conversion rate of unresectable to resectable disease is $15-20 \%$, with a 5-year OS rate of roughly $50 \%$. No definitive evidence has been published in the medical literature to indicate which modality is the best for downstaging tumors in patients with unresectable HCC. We used HAIC as the downstaging modality because HAIC can deliver high concentrations of anti-cancer agents directly to the tumor and it also minimizes systemic toxicities due to first-pass effect (9). It has also been reported that HAIC is effective against HCCs with macroscopic portal vein tumor thrombosis and intrahepatic multiple tumors who had responded well to HAIC, with a MST of 31.6 months $(9,15)$. Additionally, we preferred HAIC, because TACE or yttrium-90 micro- spheres are contraindicated in patients with major portal vein tumor thrombosis, because of their potential risk of hepatic infarction caused by arterial complication (8). One problem of downstaging chemotherapy is that we cannot predict which patients will respond. However, an important role of downstaging chemotherapy might be the selection of patients who do not progress during chemotherapy, including those who 
do not develop distant metastasis. These patients are ideal candidates for surgery due to having tumors with a lower malignant potential.

\section{Conclusion}

Salvage surgery after successful downstaging using HAIC can provide excellent outcomes, after selecting patients that are ideal surgical candidates, especially in patients with Vp3/4 and those without MVI.

\section{Conflicts of Interest}

All Authors have no conflicts of interest to disclose in relation to this study.

\section{Authors' Contributions}

Yuichi Goto designed the study, and wrote the initial draft of the manuscript. Yuichi Goto and Toru Hisaka contributed to analysis and interpretation of data, and assisted in the preparation of the manuscript. All other Authors have contributed to data collection and interpretation, and critically reviewed the manuscript.

\section{References}

1 Meric F, Patt YZ, Curley SA, Chase J, Roh MS, Vauthey JN and Ellis LM: Surgery after downstaging of unresectable hepatic tumors with intra-arterial chemotherapy. Ann Surg Oncol 7: 490495, 2000. PMID: 10947016. DOI: 10.1007/s10434-000-0490-2

2 Lee HS, Choi GH, Choi JS, Kim KS, Han K-H, Seong J, Ahn SH, Kim DY, Park JY, Kim SU and Kim BK: Surgical resection after down-staging of locally advanced hepatocellular carcinoma by localized concurrent chemoradiotherapy. Ann Surg Oncol 21: 36463653, 2014. PMID: 24916746. DOI: 10.1245/s10434-014-3652-3

3 Lee IJ, Kim JW, Han KH, Kim JK, Kim KS, Choi JS, Park YN and Seong J: Concurrent chemoradiotherapy shows long-term survival after conversion from locally advanced to resectable hepatocellular carcinoma. Yonsei Med J 55: 1489-1497, 2014. PMID: 25323884. DOI: 10.3349/ymj.2014.55.6.1489

4 Iñarrairaegui M, Pardo F, Bilbao JI, Rotellar F, Benito A, D’ Avola D, Herrero JI, Rodriguez M, Martí P, Zozaya G, Dominguez I, Quiroga J and Sangro B: Response to radioembolization with yttrium-90 resin microspheres may allow surgical treatment with curative intent and prolonged survival in previously unresectable hepatocellular carcinoma. Eur J Surg Oncol 38: 594-601, 2012. PMID: 22440743. DOI: 10.1016/j.ejso.2012.02.189

5 Warner WA, Sanchez R, Dawoodian A, Li E and Momand J: Modified Cisplatin/Interferon $\alpha-2 b /$ Doxorubicin/Fluorouracil (PIAF) chemotherapy in patients with no hepatitis or cirrhosis is associated with improved response rate, resectability and survival of initially unresectable hepatocellular carcinoma. Cancer 80: 631-637, 2013. PMID: 21959306. DOI: 10.1111/j.1747-0285.2012.01428.x.Identification

6 Song DS, Nam SW, Bae SH, Kim JD, Jang JW, Song MJ, Lee SW, Kim HY, Lee YJ, Chun HJ, You YK, Choi JY and Yoon SK: Outcome of transarterial chemoembolization-based multi-modal treatment in patients with unresectable hepatocellular carcinoma.
World J Gastroenterol 21: 2395-2404, 2015. PMID: 25741147. DOI: $10.3748 /$ wjg.v21.i8.2395

7 Zhang Y, Huang G, Wang Y, Liang L, Peng B, Fan W, Yang J, Huang Y, Yao W and Li J: Is salvage liver resection necessary for initially unresectable hepatocellular carcinoma patients downstaged by transarterial chemoembolization? Ten years of experience. Oncologist 21: 1442-1449, 2016. DOI: 10.1634/ theoncologist.2016-0094

8 Lau W, Ho SKW, Yu SCH, Lai ECH, Liew C and Leung TWT: Salvage surgery following downstaging of unresectable hepatocellular carcinoma. Ann Surg 240: 299-305, 2004. PMID: 15273555. DOI: 10.1097/01.sla.0000133123.11932.19

9 Ando E, Tanaka M, Yamashita F, Kuromatsu R, Yutani S, Fukumori K, Sumie S, Yano Y, Okuda K and Sata M: Hepatic arterial infusion chemotherapy for advanced hepatocellular carcinoma with portal vein tumor thrombosis: Analysis of 48 cases. Cancer 95: 588-595, 2002. PMID: 12209752. DOI: 10.1002/cncr.10694

10 Therasse P, Therasse P, Arbuck SG, Arbuck SG, Eisenhauer E a., Eisenhauer E a., Wanders J, Wanders J, Kaplan RS, Kaplan RS, Rubinstein L, Rubinstein L, Verweij J, Verweij J, Van Glabbeke M, Van Glabbeke M, van Oosterom AT, van Oosterom AT, Christian MC, Christian MC, Gwyther SG and Gwyther SG: New guidelines to evaluate the response to treatment in solid tumors. European Organization for Research and Treatment of Cancer, National Cancer Institute of the United States, National Cancer Institute of Canada. J Natl Cancer Inst 92: 205-216, 2000. PMID: 10655437. DOI: 10.1093/JNCI/92.3.205

11 Cherqui D, Malassagne B, Colau PI, Brunetti F, Rotman N and Fagniez PL: Hepatic vascular exclusion with preservation of the caval flow for liver resections. Ann Surg 230: 24-30, 1999. PMID: 10400032. DOI: 10.1097/00000658-199907000-00004

12 Wakayama K, Kamiyama T, Yokoo H, Kakisaka T, Kamachi H, Tsuruga Y, Nakanishi K, Shimamura T, Todo S and Taketomi A: Surgical management of hepatocellular carcinoma with tumor thrombi in the inferior vena cava or right atrium. World J Surg Oncol 11: 259, 2013. PMID: 24093164. DOI: 10.1186/1477-781911-259

13 Chun YH, Ahn SH, Park JY, Kim DY, Han KH, Chon CY, Byun SJ and Kim SU: Clinical characteristics and treatment outcomes of hepatocellular carcinoma with inferior vena cava/heart invasion. Anticancer Res 31: 4641-4646, 2011. PMID: 22199343.

14 Tang ZY, Yu YQ, Zhou X Da, Ma ZC, Yang BH, Lin ZY, Lu JZ, Liu K Da, Fan Z and Zeng ZC: Treatment of unresectable primary liver cancer: With reference to cytoreduction and sequential resection. World J Surg 19: 47-52, 1995. DOI: 10.1007/BF00316979

15 Okuda K, Tanaka M, Shibata J, Ando E, Ogata T, Kinoshita H, Eriguchi N, Aoyagi S and Tanikawa K: Hepatic arterial infusion chemotherapy with continuous low dose administration of cisplatin and 5-fluorouracil for multiple recurrence of hepatocellular carcinoma after surgical treatment. Oncol Rep 6: 587-591, 1999. PMID: 10203596. DOI: 10.3892/or.6.3.587

Received May 23, 2020

Revised June 14, 2020

Accepted June 15, 2020 